\title{
Bringing an Accurate Fresnel to Real-Time Rendering: a Preintegrable Decomposition
}

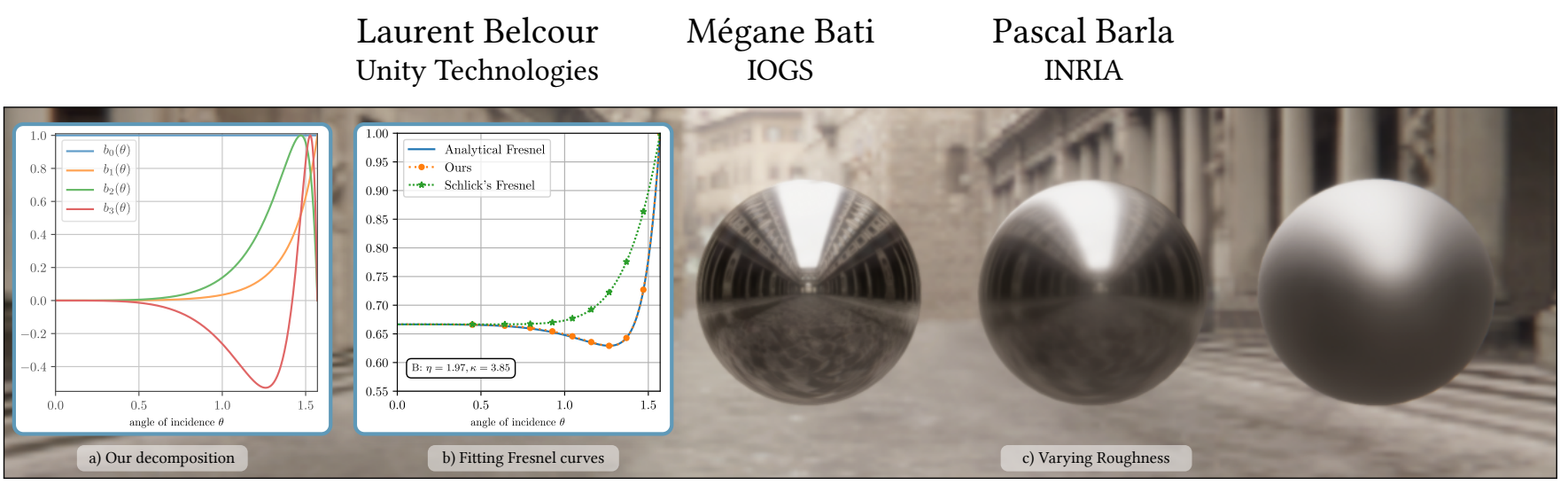

Figure 1: Our new decomposition (a) expresses Fresnel reflectance as a weighted sum of the first four eigenvectors of analytical Fresnel reflectances. They enable us to accurately represent such reflectances, even for complex metals such as Beryllium that Schlick's approximation fails to reconstruct (b). Our decomposition is compatible with image based lighting and area-light preintegration for real-time rendering. We demonstrate it in our implementation in the Unity engine (c).

\begin{abstract}
We introduce a new approximate Fresnel reflectance model that enables the accurate reproduction of ground-truth reflectance in real-time rendering engines. Our method is based on an empirical decomposition of the space of possible Fresnel curves. It is compatible with the preintegration of image-based lighting and area lights used in real-time engines. Our work permits to use a reflectance parametrization [Gulbrandsen 2014] that was previously restricted to offline rendering.
\end{abstract}

\section{CCS CONCEPTS}

- Computing methodologies $\rightarrow$ Reflectance modeling.

ACM Reference Format:

Laurent Belcour, Mégane Bati, and Pascal Barla. 2020. Bringing an Accurate Fresnel to Real-Time Rendering: a Preintegrable Decomposition. In Special Interest Group on Computer Graphics and Interactive Techniques Conference Talks (SIGGRAPH '20 Talks), August 17, 2020. ACM, New York, NY, USA, 2 pages. https://doi.org/10.1145/3388767.3407325

\section{INTRODUCTION}

VFX productions are increasingly relying on video-game engines during production instead of offline engines. To ensure coherency between the real-time and offline pipeline, coherency between their respective shading models is mandatory. Real-time and offline pipelines have converged to use microfacet reflectance models,

Permission to make digital or hard copies of part or all of this work for personal or classroom use is granted without fee provided that copies are not made or distributed for profit or commercial advantage and that copies bear this notice and the full citation on the first page. Copyrights for third-party components of this work must be honored. For all other uses, contact the owner/author(s).

SIGGRAPH '20 Talks, August 17, 2020, Virtual Event, USA

(C) 2020 Copyright held by the owner/author(s).

ACM ISBN 978-1-4503-7971-7/20/08

https://doi.org/10.1145/3388767.3407325 yet the treatment of the Fresnel reflectance that describes the interaction of light with a planar surface is not the same and leads to noticeable differences. Offline engines can afford to use the accurate Fresnel equations and currently rely on the Gulbrandsen parametrization [Gulbrandsen 2014] (see Arnold's Uber Shader for example [Georgiev et al. 2019]). Real-time engines on the other hand cannot afford the cost of the analytical Fresnel equation and rely on approximate models such as the one of Schlick [1994], the current the state-of-the-art model for video-games engines.

In this work, we introduce an alternative to Schlick's model [1994] that more accurately reproduces the ground truth Fresnel reflectances for a wide variety of surface parameters. Our solution decomposes Fresnel reflectances using four basis elements that are compatible with state-of-the-art pre-integration methods and incur a small computational overhead.

\section{FRESNEL PRE-INTEGRATION}

Real-Time Rendering Formulation. To achieve realism, modern real-time engines solve the direct illumination equation:

$$
L_{o}\left(\omega_{o}\right)=\int_{\Omega} \rho\left(\omega_{i}, \omega_{o}\right) L\left(\omega_{i}\right)\left\langle\omega_{i} \cdot \mathbf{n}\right\rangle \mathrm{d} \omega_{i}
$$

where $L_{o}$ is the outgoing radiance defined for outgoing directions $\omega_{o}, L$ the incident radiance defined over incoming directions $\omega_{i}$, and $\rho\left(\omega_{i}, \omega_{o}\right)=\frac{F\left(\omega_{i} \cdot \mathbf{h}\right) G_{1}\left(\omega_{i}, \omega_{o}\right) D_{\mathcal{v}}\left(\omega_{i}, \mathbf{h}\right)}{4\left\langle\omega_{o} \cdot \mathbf{h}\right\rangle}$ is the microfacet-based BRDF model with $\mathbf{h}=\frac{\omega_{i}+\omega_{o}}{\left\|\omega_{i}+\omega_{o}\right\|}$ with a visible normal distribution $D_{v}(\mathbf{h})$, shadowing term $G_{1}\left(\boldsymbol{\omega}_{i}, \boldsymbol{\omega}_{o}\right)$, and Fresnel term $F\left(\boldsymbol{\omega}_{i} \cdot \mathbf{h}\right)$. To achieve real-time frame rates, this equation is approximated by splitting the integral into two terms $L_{o}\left(\omega_{o}\right) \simeq \bar{F}\left(\omega_{o}\right) \times \bar{L}\left(\omega_{o}\right)$ [Lagarde and De Rousiers 2014]. $\bar{F}$ includes the average material response with respect to the distribution of visible normals, and $\bar{L}$ the average 
light response:

$$
\begin{aligned}
& \bar{L}\left(\omega_{o}\right)=\int_{\Omega} L\left(\omega_{i}\right) D_{v}\left(\omega_{i}, \mathbf{h}\right) \mathrm{d} \omega_{i}, \\
& \bar{F}\left(\omega_{o}\right)=\int_{\Omega} \frac{F\left(\omega_{i} \cdot \mathbf{h}\right) G_{1}\left(\omega_{i}, \omega_{o}\right) D_{v}\left(\omega_{i}, \mathbf{h}\right)}{4\left\langle\omega_{o} \cdot \mathbf{h}\right\rangle} \mathrm{d} \omega_{i} .
\end{aligned}
$$

Both $\bar{L}$ and $\bar{F}$ are tabulated with respect to their parameters: $\bar{L}$ depends on the roughness of the $v n d f$ and the mean reflected direction; $\bar{F}$ depends on $\omega_{o} \cdot \mathbf{n}$, the roughness and the complex Index of Refraction (IOR) $\boldsymbol{\eta}$. The latter is thus four dimensional.

Split-Sum Approximation. To reduce the storage cost of the material integral, Karis introduced the Split-Sum Integral [Karis 2013] that uses Schlick's decomposition of the Fresnel term:

$$
\bar{F} \simeq F_{0} \overline{S_{0}}+\left(1-F_{0}\right) \overline{S_{1}},
$$

where $\overline{S_{0}}$, and $\overline{S_{1}}$ are the average of Schlick's base functions: $s_{0}\left(\omega_{i}\right.$. $\mathbf{n})=1$, and $s_{1}\left(\omega_{i} \cdot \mathbf{n}\right)=\left(1-\omega_{i} \cdot \mathbf{n}\right)^{5}$ [Schlick 1994].

However, Schlick's equation is a poor approximation, especially for metals. Also, it is only parametrized by the reflectance at normal incidence and cannot accommodate for hue variation at grazing angles. This prevents the use of artistic control over metal reflectance [Gulbrandsen 2014] in modern real-time rendering engines (see Figure 2 right).

\section{AN EMPIRICAL DECOMPOSITION}

Fresnel Decomposition. Our work builds on decomposing of the Fresnel reflectance as a weighted sum of basis functions $b_{i}(\theta)$ :

$$
F(\theta ; \boldsymbol{\eta})=\sum c_{i}(\boldsymbol{\eta}) b_{i}(\theta)
$$

By separating degrees of freedom of the Fresnel reflectance between the base functions and the coeffcients, we provide a form amendable to the Split-Sum Integral trick. Indeed:

$$
\bar{F}=\sum c_{i}(\boldsymbol{\eta}) \overline{b_{i}},
$$

where $\overline{b_{i}}$ is the integral of $b_{i}(\theta)$ times the microfacet response.

Basis Functions. We determine $b_{i}(\theta)$ using an iterative Eigenvectors extraction (using SVD) over a large set of Fresnel reflectances to generate the different base functions. We forced $b_{0}(\theta)=1$ as the first element and shifted and rescaled the Fresnel reflectances so that the decomposed curves start at 0 and ends at 1 . At each iteration, a new basis is obtained by removing the current approximation from the ground truth reflectance, and taking the first eigen vector of its SVD:

$$
b_{k}=\operatorname{svd}\left[F-\sum_{i=0}^{k-1} c_{i} B_{i}\right]_{0},
$$

where svd [. $]_{0}$ is the first eigenvector. Figure 1 (a) shows the first four elements of our decomposition. In practice, we only kept 4 elements in our basis: the Split-Sum preintegration requires a texture channel per basis element. Four basis elements fits a RGBA texture, requiring a single fetch at runtime.
Coefficients. We evaluate the coefficients of this decomposition in the same iterative way by evaluating the Fresnel curve at specific positions. When using 4 basis elements, at most 4 evaluations of the target Fresnel curve are required to constrain the system of unknowns. For that, we used $\theta_{0}=0, \theta_{1}=\frac{\pi}{2}$ to fully determine $c_{0}$ and $c_{1}$, and we use $\theta_{2} \simeq 1.45, \theta_{3} \simeq 1.31$ to determine $c_{2}$ and $c_{3}$. See our supplemental materials for more details on fitting.

Gulbrandsen's Parametrization. Since the artistic parametrization of metals of Ole Gulbrandsen [2014] enables to evaluate the exact Fresnel curve using the reflectivity $r$ and edgetint $g$, it is possible to extract $c_{0}, c_{1}, c_{2}, c_{3}$ from $r$ and $g$ using the method already described.

\section{RESULTS}

We implemented our Fresnel decomposition in an offline path tracer, a real-time prototype using OpenGL and in the Unity engine. See our supplemental materials and video for more results.

Figure 1 (b), show the integration of our decomposition for a Beryllium conductor in Unity. In Figure 2, we show in our OpenGL prototype that our method correctly reproduces the edgetint of Gulbrandsen. There, we measured that our method was at most 0.05 ms slower than Schlick's decomposition in full screen at 720p on an Nvidia RTX 2070.
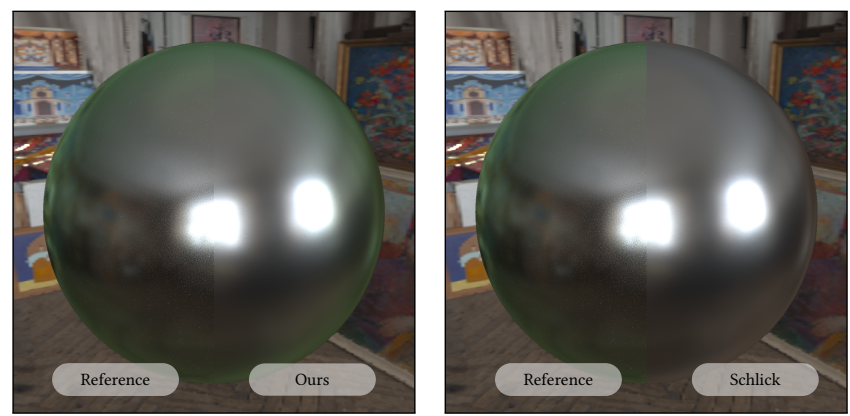

Figure 2: Our method reproduces better the edgetint than Schlick's decomposition compared to a Monte-Carlo reference. We used $r=[0.44,0.44,0.44], g=[0,1,0]$ and $\alpha=0.08$.

\section{ACKNOWLEDGMENTS}

The authors thank Thomas Deliot for implementing the method in the Unity engine and Kenneth Vanhoey, Jonathan Dupuy and Eric Heitz for proof-reading.

\section{REFERENCES}

Iliyan Georgiev, Jamie Portsmouth, Zap Andersson, Adrien Herubel, Alan King, Shinji Ogaki, and Frederic Servant. 2019. Arnold's Uber Shader. https://autodesk.github. io/standard-surface/\#closures/metal.

Ole Gulbrandsen. 2014. Artist Friendly Metallic Fresnel. Journal of Computer Graphics Techniques (FCGT) 3, 4 (9 December 2014), 64-72.

Stephen Hill. 2019.2 A Multi-Faceted Exploration. https://blog.selfshadow.com/2018/05/13/multi-faceted-part-1/.

Brian Karis. 2013. Real Shading in Unreal Engine 4. In Physically Based Shading in Theory and Practice - SIGGRAPH Courses.

Sebastien Lagarde and Charles De Rousiers. 2014. Moving Frostbite to PBR. In SIGGRAPH Course - Physically Based Shading Theory Practice.

Christophe Schlick. 1994. An inexpensive BRDF model for physically-based rendering. In Computer graphics forum, Vol. 13. Wiley Online Library, 233-246. 